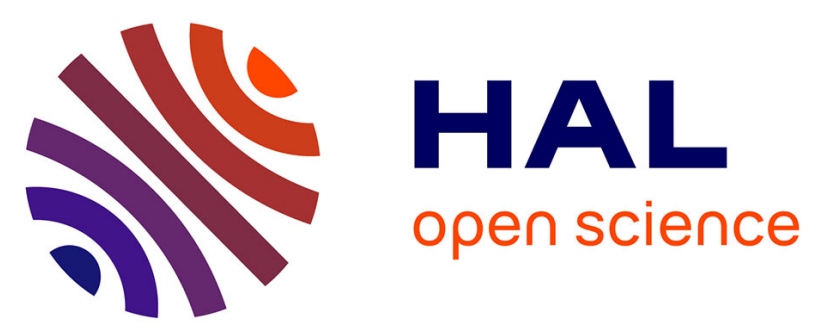

\title{
Long-term Visual Outcomes and Causes of Vision Loss in Chronic Central Serous Chorioretinopathy
}

Sarah Mrejen, Chandrakumar Balaratnasingam, Talia R. Kaden, Alexander Bottini, Kunal Dansingani, Kavita V. Bhavsar, Nicolas A. Yannuzzi, Samir Patel, Kevin C. Chen, Suqin Yu, et al.

\section{To cite this version:}

Sarah Mrejen, Chandrakumar Balaratnasingam, Talia R. Kaden, Alexander Bottini, Kunal Dansingani, et al.. Long-term Visual Outcomes and Causes of Vision Loss in Chronic Central Serous Chorioretinopathy. Ophthalmology: Journal of The American Academy of Ophthalmology, 2019, 126, pp.576 - 588. 10.1016/j.ophtha.2018.12.048 . hal-03486015

\section{HAL Id: hal-03486015 https://hal.science/hal-03486015}

Submitted on 20 Dec 2021

HAL is a multi-disciplinary open access archive for the deposit and dissemination of scientific research documents, whether they are published or not. The documents may come from teaching and research institutions in France or abroad, or from public or private research centers.
L'archive ouverte pluridisciplinaire HAL, est destinée au dépôt et à la diffusion de documents scientifiques de niveau recherche, publiés ou non, émanant des établissements d'enseignement et de recherche français ou étrangers, des laboratoires publics ou privés.

\section{()ㅜ(1)}

Distributed under a Creative Commons Attribution - NonCommercial| 4.0 International 
Title: Long-term Visual Outcomes and Causes of Vision Loss in Chronic Central Serous Chorioretinopathy

\section{Short title: Long-term visual outcomes in CSC}

\section{Authors and Affiliations:}

Sarah Mrejen, $\mathrm{MD}^{1,2,3}$; Chandrakumar Balaratnasingam, MD, $\mathrm{PhD}^{1,2,4,5,6}$; Talia R. Kaden, $\mathrm{MD}^{1,2,4,7}$; Alexander Bottini, MD ${ }^{4}$; Kunal Dansingani, MD ${ }^{1,2,8}$; Kavita V. Bhavsar, MD ${ }^{1,2,9,10}$; Nicolas A. Yannuzzi, MD ${ }^{11}$; Samir Patel, MD ${ }^{12}$; Kevin C. Chen, MD ${ }^{4,13}$; Suqin Yu, MD ${ }^{1,2,14}$; Guillaume Stoffels $\mathrm{PhD}^{15}$; Richard F. Spaide, $\mathrm{MD}^{1,2} ; \mathrm{K}$. Bailey Freund, MD ${ }^{1,2,4}$; Lawrence A. Yannuzzi, MD ${ }^{1,2,4}$

${ }^{1}$ Vitreous, Retina, Macula Consultants of New York, NY, USA

${ }^{2}$ LuEsther T. Mertz Retinal Research Center, Manhattan Eye, Ear, and Throat Hospital, New York, NY, USA

${ }^{3}$ Quinze-Vingts Hospital, DHU SightMaintain, INSERM-DHOS CIC 1423, Paris, France

${ }^{4}$ Department of Ophthalmology, New York University School of Medicine, New York, NY, USA

${ }^{5}$ Department of Physiology and Pharmacology, Centre for Ophthalmology and Visual Science, Lions Eye Institute, University of Western Australia, Perth, Australia.

${ }^{6}$ Department of Ophthalmology, Sir Charles Gairdner Hospital, Perth, Australia

${ }^{7}$ Department of Ophthalmology, Manhattan Eye Ear and Throat Hospital, New York, NY, USA

${ }^{8}$ Department of Ophthalmology, University of Pittsburgh Medical Center, Pittsburgh, Pennsylvania, USA

${ }^{9}$ Casey Eye Institute, Oregon Health and Science University, Portland, Oregon, USA

${ }^{10}$ Portland VA Healthcare System, Oregon, USA

${ }^{11}$ Department of Ophthalmology, Bascolm Palmer Eye Institute, Miami, Florida, USA

${ }^{12}$ Department of Ophthalmology, Wills Eye Hospital, Philadelphia, Pennsylvania, USA

${ }^{13}$ Vantage Eye Center, Salinas, California, USA

${ }^{14}$ Department of Ophthalmology, Shanghai General Hospital, Shanghai, China

${ }^{15}$ Biostatistics Unit of Feinstein Institute for Medical Research, NY, USA

\section{Corresponding author:}

Dr. Sarah Mrejen, MD

Vitreous Retina Macula Consultants of New York

460 Park Avenue, Fifth Floor, New York, NY 10022

Telephone: 212-861-9797

Fax: 212-628-0698

(E): sarahmrejen.uretsky@gmail.com

This article contains additional online-only material: Supplementary Tables 1, 2 and 3.

\section{Meeting Presentation: No}


43 Financial Support: LuEsther T. Mertz Retinal Research Center, Manhattan Eye, Ear and

Throat Hospital, New York, NY, USA, and The Macula Foundation, Inc., New York, NY, USA. The funding organizations had no role in the design or conduct of this research.

Conflict of Interest/Disclosures: S Mrejen is a consultant to Novartis and Bayer. Chandrakumar Balaratnasingam is a consultant to Novartis, Bayer and Allergan. L Yannuzzi receives an honorarium from Genentech for the retina fellow teaching program. K B Freund is a consultant to Genentech, Optos, Optovue, Heidelberg Engineering, and Graybug Vision, and receives research support from Genentech//Roche. Richard F. Spaide receives consulting and royalty payments from Topcon Medical Systems, royalties from DORC, and consulting fees from Heidelberg Inc. The other authors have no conflicting interests to disclose.

Manuscript word Count: 6378 words

\section{Key Words:}

Central Serous Chorioretinopathy;

Choroidal Neovascularization;

Cystoid Macular Degeneration;

Foveal Atrophy;

Fundus Autofluorescence Imaging;

Macular Degeneration;

Multimodal Imaging;

Spectral Domain Optical Coherence Tomography;

Visual Prognosis 
67

\section{$\underline{\text { ABSTRACT }}$}

PURPOSE: To evaluate the long-term visual outcomes and causes of vision loss in chronic central serous chorioretinopathy (CSC).

DESIGN: Retrospective, longitudinal study

SUBJECTS: One-hundred and thirty-three subjects (217 eyes) with chronic CSC.

METHODS: A retrospective review of clinical and multimodal imaging data of patients with chronic CSC managed by 3 of the authors between May 1977 and March 2018. Multimodal imaging comprised color photography, fluorescein angiography, indocyanine green angiography, fundus autofluorescence (FAF) and optical coherence tomography (OCT).

MAIN OUTCOME MEASURES: Best corrected visual acuity (BCVA) at the final visit; change in BCVA between first visit and 1, 5 and 10-year follow-up visits, and causes of vision loss at final visit.

RESULTS: Data from 6,228 individual clinic visits were analyzed. Mean age of patients at the first visit was 60.7 years and mean period of follow-up from first to last visit was 11.3 years. The cohort included 101 males (75.9\%). At the final visit, 106 patients $(79.7 \%)$ maintained driving-standard vision with BCVA of 20/40 or better in at least one eye and 17 patients $(12.8 \%)$ were legally blind with BCVA of 20/200 or worse in both eyes. Mean BCVA at first visit was not significantly different from mean BCVA at 1 or 5 -year follow-up visits (both $\mathrm{p} \geq 0.65$ ) but was significantly better than the mean BCVA at the 10-year follow-up visit ( $\mathrm{p}=0.04)$. Seventynine percent of eyes with 20/40 or better vision at the first visit maintained the same level of vision at the 10-year follow-up visit. Ninety-two percent of eyes with 20/200 or worse vision at 
88 the first visit maintained the same level of vision at the 10-year follow-up visit. Cystoid macular degeneration, choroidal neovascularization, outer retinal disruption on OCT and FAF changes were associated with poorer vision at final visit (all $\mathrm{p} \leq 0.001$ ). Multivariable analysis revealed $\operatorname{visit}(\mathrm{p}=0.001)$.

93 CONCLUSION: Chronic CSC can be a sight-threatening disease leading to legal blindness. Age at presentation and outer retinal changes on multimodal imaging were associated with longterm BCVA changes and may be predictors of long-term visual outcomes. 


\section{Précis}

101 Visual outcomes in chronic CSC are generally favorable but $12.8 \%$ of patients were legally blind 102 after 10 years in a retrospective study population in a referral center for chronic CSC. A

103 statistically significant determinant of long-term visual change was a greater age at first visit. 


\section{INTRODUCTION}

Central serous chorioretinopathy (CSC) is a complex disorder of unresolved etiology that is characterized by detachments of the retinal pigment epithelium (RPE) and neurosensory retina. ${ }^{1}$ The initial manifestations of this disease are most frequently observed between 20 and 50 years of age. ${ }^{2,3}$ As CSC predominantly affects the working age population, vision loss due to this condition can lead to significant reduction in patient independence, greater loss of productive work years and a larger cumulative toll on health services. Despite a plethora of investigative reports that span nearly 150 years, our understanding of the long-term visual and anatomic outcomes in CSC remain confined to only a few studies of limited sample size and follow up. ${ }^{3-6}$ Expanding our understanding of these key areas has major relevance for patient education, refining management paradigms and rationalizing medical services.

The natural course of CSC is significantly heterogeneous and highly varied between individuals. ${ }^{7}$ The anatomic sequelae of CSC, with respect to the magnitude of retina and RPE injury, is plausibly related to the morphologic characteristics and chronicity of neurosensory and RPE detachments. Prolonged separation of photoreceptors from the RPE and choroid, due to persistent subretinal fluid, may result in hypoxic injury to the outer retina. ${ }^{8}$ Altered hydrostatic pressures and vascular permeability within the choroidal circulation may also induce irreversible structural changes to the RPE. The chronic variant of CSC is characterized by persistent neurosensory detachments and/or a natural course that is marred by recurrences. ${ }^{1,9}$ The protracted course and relapsing-remitting nature of chronic CSC is therefore likely to confer a greater risk of vision loss than the self-limiting variant commonly referred to as acute CSC. Visual outcomes in acute CSC have been extensively documented ${ }^{10}$ however the postulation that 
chronic CSC portends a relatively poorer visual prognosis is yet to be evaluated in a systematic manner.

The risk factors for $\mathrm{CSC}^{11-13}$ and the phenotypic manifestations of the chronic variant including pigment epithelial detachments (PEDs), ${ }^{14,15}$ choroidal thickening, ${ }^{16,17}$ gravitating atrophic tracts ${ }^{18}$ and RPE abnormalities ${ }^{19-23}$ have been described in depth. The complications that can occur during the natural course of chronic CSC such as choroidal neovascularization (NV), ${ }^{24}$ cystoid macular degeneration (CMD) ${ }^{25}$ and foveal atrophy ${ }^{21}$ have also been clearly defined. What is lacking is information concerning the relationship between the risk factors for CSC, the clinical features of this disease and long-term visual outcomes. In this longitudinal study, we examined the visual outcomes in 217 eyes of 133 patients with chronic CSC and sought to define the major demographic and clinical factors associated with long-term vision loss.

\section{METHODS}

This study received approval by the Western Institutional Review Board (Olympia, WA, USA). It complied with the Health Insurance Portability and Accountability Act of 1996 and followed the tenets of the Declaration of Helsinki.

\section{$\underline{\text { SUBJECTS }}$}

We retrospectively reviewed the charts and imaging data for patients diagnosed with chronic CSC by 3 retina specialists (LAY, KBF, RFS) at the private offices of Vitreous, Retina, Macula Consultants of New York (VRMNY). All patients were examined between May 1977 and March 2018.

Subjects were included in the study if they were diagnosed with a neurosensory detachment attributed to one or more sites of leakage at the level of the RPE at the first clinic visit and if there was documentation of persistent subretinal fluid on consecutive visits that spanned 6 or 
more months. ${ }^{1}$ Exclusion criteria included: (i) neurosensory detachment due to diseases other than CSC, (ii) severe media opacities, (iii) severe glaucoma, (iv) history of rhegmatogenous retinal detachment, trauma, or intraocular inflammation, (v) angioid streaks, (vi) pathologic myopia.

For every patient, demographic information including age at first clinic visit, age at last clinic visit, gender and race was recorded. Medical information including smoking status, history of hypertension, diabetes and corticosteroid use was also collected. Information regarding best corrected visual acuity (BCVA), refractive error, ocular co-morbidities and previous treatments for CSC were also determined for each visit using patient notes. When relevant, we also made a distinction between age at $1^{\text {st }}$ visit to VRMNY (henceforth referred to as 'age at $1^{\text {st }}$ visit') and age of onset of disease. For example, some patients were under the care of other ophthalmologists prior to attending VRMNY and therefore the age of disease onset, as determined using documents from referrers, was different from the age at $1^{\text {st }}$ visit.

\section{MULTIMODAL IMAGING AND IMAGE ANALYSIS}

The clinical course of CSC was studied using a combination of color photography, fundus autofluorescence (FAF), dye angiography (fluorescein and/or indocyanine green) and optical coherence tomography. Imaging was performed using technology that was available at the time of the visit. All patients had at least one visit during their disease course where spectral-domain optical coherence tomography (SD-OCT) and FAF imaging were both acquired.

Color photographs, FAF, fluorescein angiography (FA) and indocyanine green angiography (ICGA) images were obtained using the Optos 200Tx (Optos 200Tx; Optos, Dunfermline, Scotland, United Kingdom) and/or Topcon TRC 501X fundus camera (Topcon Imagenet, Tokyo, Japan) and/or Heidelberg Spectralis HRA+OCT (Heidelberg Engineering, Germany). The 
peripheral retina was evaluated using Optos ultra-widefield imaging or by montaging Topcon color images.

Macular OCT evaluations were performed using the Heidelberg Spectralis HRA+OCT (Heidelberg Engineering, Germany; volume scan between $20^{\circ} \times 15^{\circ}$ and $30^{\circ} \times 25^{\circ}$ in dimensions). Some eyes were also imaged with time-domain OCT (Stratus, Carl Zeiss Meditec Inc., Dublin, California). Enhanced depth imaging OCT scans (Heidelberg Engineering, Germany) over a $30^{0} \times 5^{0}$ degree region of the central macula, with 100 images averaged using 7 sections, were used to evaluate choroidal features. Subfoveal choroidal thickness was also measured using SDOCT and was defined as the distance between the RPE-Bruch's membrane complex and the choroid-scleral border under the fovea.

Multimodal imaging data was used to determine laterality of disease and to determine the occurrence of the following:

- Choroidal neovascularization - Diagnosed using dye angiography (fluorescein and/or indocyanine green). ${ }^{3,6,24,26}$ Angiographic features suggestive of NV included visualization of a neovascular network in the early phase of FA or ICGA, leakage on FA and a hyperfluorescent plaque on ICGA that was most clearly evident in the late frames of the angiogram (Figure 1). Optical coherence tomography (when available) was also used to aid the diagnosis of NV and lesions were classified into type 1,2 and 3 lesions using the Gass-Freund classification. ${ }^{27-29}$ The location of NV was determined according to the Macular Photocoagulation Study (MPS) terminology and classified as subfoveal, juxtafoveal and extrafoveal lesions..$^{30}$

- Shallow irregular PED in the macula - SD-OCT macular volume scans from the final visit were evaluated for the presence of a shallow irregular PED as previously described 
by Hage et $a l .{ }^{31}$ Specifically, a shallow, irregular PED was defined as an irregular RPE profile that was separated from Bruch membrane with heterogeneous internal reflectivity.

- Retinal pigment epithelial alterations - Fundus autofluorescence imaging from the final visit was used to evaluate the RPE (Figure 2). Patterns of FAF changes were qualitatively assessed using previously published criteria ${ }^{21,23,32}$ and the total area of hypoautofluorescence in the posterior pole was measured and classified as follows: (1) Less than 2 MPS disk areas; (2) Between 2-4 MPS disk areas; (3) Greater than 4 MPS disk areas. The pattern of foveal hypoautofluorescence was also classified as confluent or granular. ${ }^{21}$ The presence or absence of descending tracts was also recorded.

- Structural alterations of the outer retina - The integrity of the ellipsoid zone (EZ) and external limiting membrane in the central fovea at the final visit was assessed using SDOCT and was graded as being disrupted or intact. The occurrence of subretinal fibrosis in any region of the retina at the final visit was also graded as being present or absent using SD-OCT, color imaging and FAF criteria.

- Cystoid macular degeneration - Graded as being present or absent in the fovea. Cystoid macular degeneration was defined as the occurrence of intraretinal cystoid spaces on OCT without intraretinal leakage on FA (Figure 3 ). ${ }^{25} \mathrm{In}$ these eyes, the central $1 \mathrm{~mm}$ subfield thickness was also recorded from the retinal thickness ETDRS grid generated by Spectralis Eye Explorer review software (Heidelberg Engineering, Heidelberg, Germany). Point thickness of the central fovea was also determined using the B-scan image of the central fovea and defined as the distance between the retinal pigment epithelium and inner limiting membrane. Calipers provided by the OCT Spectralis software were used to determine point thickness of the central fovea. 
- Bullous retinal detachment was defined as a neurosensory detachment (more than 10 disc diameters) with a bullous configuration that extended from the periphery to the inferior vascular arcades in the seated position. ${ }^{19}$

\section{STATISTICAL ANALYSIS}

For statistical analyses, BCVA was expressed in logarithm of minimum angle of resolution (logMAR) units. For some analyses, eyes were stratified into 3 groups based on BCVA [Group 1 (good BCVA) - $\log$ MAR $\leq 0.3$ (Snellen equivalent 20/40 or better); Group 2 (intermediate BCVA) $-0.3<\operatorname{logMAR}<1$ (Snellen equivalent between 20/200 and 20/40); Group 3 (poor BCVA) - $\log M A R \geq 1$ (Snellen equivalent $20 / 200$ or worse)). At each follow-up time point (i.e. 1-year, 5-year, or 10-year follow-up) patient-level baseline categorical variables were compared between selected and not selected eyes using the Chi-square or Fisher's exact test (as appropriate), and eye-level continuous variables were compared between the 2 groups using simple marginal linear models that accounted for inter-eye correlations as shown in Supplementary Table $1 .{ }^{33}$ For example, if a patient did not have 5-year follow up data but had available 10-year follow up data they would appear in the not selected group for the 5-year analysis and the selected group for the 10-year analysis. To avoid using multiple tables, all variables were reported at the eye level. To assess whether BCVA changed between baseline (i.e. first visit) and each follow-up time point, a marginal model was used for each follow-up time point, where the difference in BCVA was expressed as a function of a constant term (i.e. intercept). To identify factors of BCVA change, we used marginal models that expressed BCVA at follow-up as a function of baseline BCVA and one (univariable analyses) or several other baseline/treatment variables (multivariable analysis). Univariable and multivariable analyses were repeated for each of the 3 follow-up time points. In multivariable analyses, variable 
selection was accomplished using Hosmer and Lemeshow's "Purposeful Selection of

Covariates" selection algorithm adapted to linear models. ${ }^{34}$ For variables measured only at last visit (i.e. FAF changes at central fovea, subfoveal choroidal thickness, cystoid macular edema, photoreceptor lines disruption, and external limiting membrane disruption at fovea) or at unknown time before last visit (i.e. CNV), we did a cross-sectional data analysis that assessed the association between BCVA at last visit and each variable using simple linear marginal models.

A result was considered statistically significant at the $p<0.05$ level of significance. All analyses were performed using SAS version 9.4 (SAS Institute, Cary, NC).

\section{RESULTS}

\section{GENERAL}

252 A total of 217 eyes of 133 subjects met the inclusion criteria for this study. Data from 6,228 individual clinic visits were collected and analyzed. The demographic and clinical features of the cohort are summarized in Table 1. The cohort included 101 males (75.9\%) and $104(47.9 \%)$ left eyes. Eighty-four subjects (63.2\%) had bilateral chronic CSC. Mean age of onset was $53.7 \pm$ 11.9 years $($ median $=53.0$ years, minimum $=29.2$ years and maximum $=85.0$ years $)$. The mean age at first clinic visit was $60.7 \pm 11.0$ years $($ median $=60.3$ years, minimum $=37.7$ years and maximum $=90$ years) and mean age at final clinic visit was $69.3 \pm 9.4$ years (median $=68.0$ years, minimum $=55.0$ years and maximum $=97.0$ years $).$ The mean duration of follow-up from onset to last visit was $18.3 \pm 11.3$ years (median 17.2 years, minimum $=24.2$ weeks and maximum $=48.4$ years $)$. The majority of the patients self-identified as white $(119 ; 89.5 \%)$. Thirteen $(9.8 \%)$ had a history of diabetes mellitus, $43(32.3 \%)$ had a history of systemic hypertension and $27(20.5 \%)$ patients were smokers. There was a history of corticosteroid use 
either orally, parentally or via inhalation in 33 patients (24.8\%). Five eyes developed bullous neurosensory detachment during their clinical course that resolved completely with treatment.

Mean BCVA at the first visit was $0.43 \pm 0.47 \log$ MAR (Snellen equivalent 20/56) (median $=$ $0.30 \log \mathrm{MAR}$, minimum $=-0.12 \log \mathrm{MAR}$ and maximum $=1.90 \log \mathrm{MAR})$ and at the final visit was $0.52 \pm 0.55 \log$ MAR (Snellen equivalent 20/66) $($ median $=0.30 \log$ MAR, minimum $=-0.12$ $\log$ MAR and maximum $=3.0 \log$ MAR). At the final visit, 106 patients $(79.7 \%)$ had $\log$ MAR VA $\geq 0.3$ in at least one eye (Snellen equivalent $20 / 40$ or better) and 17 patients $(12.8 \%)$ had $\log$ MAR VA $\leq 1$ in both eyes (Snellen equivalent 20/200 or worse).

Refraction was available for 189 eyes $(87.1 \%)$. Ninety-two eyes $(48.7 \%)$ were hyperopic (range +1.00 to $+5.50 \mathrm{D}), 56$ eyes $(29.6 \%)$ were emmetropic (range $0.0 \pm 0.75 \mathrm{D})$ and 41 eyes $(21.7 \%)$ were myopic (range $-1.0 \mathrm{D}$ to $-6.0 \mathrm{D}$ ). The mean refractive error was 0.16 diopters.

Overall, one hundred and thirteen eyes (52.1\%) were treated with PDT, thermal laser, or antiVEGF therapy; the remaining 104 eyes received no treatment. Seventy-one (32.7\%) eyes were treated with PDT, of which 31 eyes $(14.3 \%)$ received PDT alone. Of the eyes that received PDT, $40(18.4 \%)$ had only one PDT session. Sixty-eight (31.3\%) eyes were treated with anti-VEGF injections, slightly less than half of which $(29 ; 13.4 \%)$ were treated only with anti-VEGF injections. Twenty eyes $(9.2 \%)$ were treated with thermal laser, $11(5.1 \%)$ of which received laser alone. Four eyes (1.8\%) were treated with all three treatment modalities. The most common treatment for any eye, including both eyes that received monotherapy and combination therapy, was a combination of PDT with anti-VEGF injections $(33 ; 15.2 \%)$. This combination was also by far the most common bimodal therapy (78.6\%).

\section{CHOROIDAL NEOVASCULARIZATION AND STRUCTURAL ALTERATIONS OF}


The findings for this section are summarized in Table 2. Fifty-two eyes (24.0\%) of 37 patients were complicated by neovascularization, all of which were classified as type $1 \mathrm{NV}$. An example of the typical clinical features of type $1 \mathrm{NV}$ in a patient with chronic CSC is presented in figure 1. Type $1 \mathrm{NV}$ was diagnosed on average $17.0 \pm 10.4$ years after the first clinic visit $($ median $=$ 18.0 years, minimum $=2.0$ years and maximum $=37.0$ years $)$ and the mean age of subjects upon diagnosis of NV was $67.7 \pm 8.3$ years $($ median $=62.4$ years, minimum $=49.3$ years and maximum $=77.8$ years). Twenty-two of the $52 \mathrm{NV}$ lesions $(42.3 \%)$ were subfoveal, 14 lesions (26.9\%) were juxtafoveal and 16 lesions (30.8\%) were extrafoveal. Mean subfoveal choroidal thickness at the final visit was $397.4 \pm 131.8 \mu \mathrm{m}$.

Fundus autofluorescence imaging from the final visit demonstrated RPE alterations in all eyes in the study. Varying morphologic patterns of FAF changes were observed, including mild hyperautofluorescent and hypoautofluorescent changes, multifocal lesions in the posterior pole and gravitating tracts extending into the inferior peripheral retina (Figure 2). Eyes that had developed bullous retinal detachment during their clinical course demonstrated widespread areas of hypoautofluorescence in the inferior retina at the site of previous detachment. The area of hypoautofluorescence in the posterior pole was less than 2 MPS disk diameters in 67 eyes diameters in 103 eyes (47.5\%). Gravitating tracts were seen in 105 eyes (48.4\%). Of the eyes with gravitating tracts, a single gravitating tract was seen in 63 eyes $(60.0 \%)$, two gravitating tracts were seen in 31 eyes $(29.5 \%)$ and 3 or more gravitating tracts were seen in 11 eyes (10.5\%). Foveal hypoautofluorescence was observed in 162 eyes $(74.7 \%)$ at the final visit. Of these, 59 eyes $(36.4 \%)$ demonstrated confluent hypoautofluorescence of the central fovea and the 
remainder (63.6\%) demonstrated granular hypoautofluorescence (Figure 2). Shallow, irregular PEDs were seen on SD-OCT in 56 eyes $(25.8 \%)$ at the final visit. Twenty-nine of these 56 eyes had CNV (51.8\%).

With regard to foveal outer retinal structure, 90 eyes (41.5\%) demonstrated disruption of the EZ at the final visit. Disruptions in the external limiting membrane band was seen in 146 eyes (67.3\%). Subretinal fibrosis was seen in 25 eyes (11.5\%) and CMD was seen in 47 eyes $(21.7 \%)$ at the final visit. An example of the typical clinical features of CMD in a patient with chronic CSC is presented in Figure 3. All eyes with CMD demonstrated RPE attenuation and atrophy at the macula, which was best visualized using color photographs and FAF imaging.

Hyperfluorescence due to window defects were seen in the early frames of the FA but no leakage was evident during the late frames of the FA or ICGA. Variable number of intraretinal cysts was seen on SD-OCT and outer retinal disruption was also evident in some eyes with CMD. Mean central subfield thickness in eyes with CMD was $436.8 \pm 257.6 \mu \mathrm{m}$ (range 130-1282) and mean point thickness of the central fovea was $451.4 \pm 420.1 \mu \mathrm{m}$ (range 76-1699).

\section{CHANGE IN BCVA BETWEEN FIRST VISIT AND 1-, 5- AND 10-YEAR FOLLOW-UP}

\section{VISITS}

\section{BCVA 1 year \pm 3 months after first visit}

There were a total of 131 eyes ( 82 patients) with at least one follow-up visit between 9 and 15 months after the first visit. Among the remaining 86 eyes in the cohort that were not selected for this analysis, 14 eyes had a duration of follow-up shorter than 9 months and 72 eyes had a duration of follow-up longer than 15 months.

Comparisons of baseline variables between selected and not selected groups are provided in Supplementary Table 1. Eyes that had a 1-year follow-up visit (+/- 3 months) were significantly 
older at $1^{\text {st }}$ visit compared to eyes that did not (mean age $=63$ vs. 57 years; $p=0.0003$ ). Also their age of disease onset was significantly greater (mean age $=55$ vs. $50 ; \mathrm{p}=0.01$ ) and they had poorer vision acuity (median $\mathrm{BCVA}=0.30$ vs. $0.18 ; \mathrm{p}=0.001$ ). There was not enough evidence to conclude that any other baseline variable differed between the two groups.

The most common treatment pattern in the selected group was 'no treatment' (78 eyes; 60\%), followed by 1 session of PDT laser (20 eyes; 15\%), 2 sessions of PDT laser (7 eyes; 5.3\%), and 1 session of thermal laser (7 eyes; 5.3\%). All other eyes (19 eyes; $14.5 \%)$ received 1-8 sessions of anti-VEGF therapy plus 0-3 sessions of PDT laser.

There was not enough evidence to conclude that mean BCVA at baseline and at 1 year differed ( 0.49 vs. 0.49 , respectively; $\mathrm{p}=0.70)$. In this group, the most common pattern was stable good vision from first visit to 1-year follow-up (36\% of eyes), followed by stable poor vision (20\%), stable intermediate vision (19\%), and improvement from intermediate to good vision (11\%) as shown in Supplementary Table 2. Most eyes with either good or poor initial vision tended to stay in their BCVA group at the 1-year visit (89\% and 79\%, respectively). However, only 56\% of eyes with intermediate vision at first visit maintained their BCVA status after 1 year, while $31 \%$ improved and $13 \%$ deteriorated.

Univariable analysis (Table 3) revealed that number of years from disease onset to first visit $(p=0.01)$ and left eye $(p=0.04)$ were significantly associated with deterioration of BCVA between initial and 1-year visits. Specifically, each additional year between onset and initial visit was associated with an increase of $0.007 \operatorname{logMAR}(95 \% \mathrm{CI}$ : 0.002 to 0.011$)$ and left vs. right eye was associated with a $0.09 \log$ MAR decrease $(95 \%$ CI: -0.17 to -0.01$)$. There was not enough evidence to conclude that any other proposed factors were associated with change in BCVA at 1 year (Table 3). 
Number of years from onset to $1^{\text {st }}$ visit $(\mathrm{p}=0.01)$ and left eye $(\mathrm{p}=0.03)$ were the only variables found to be significantly associated with BCVA change in the multivariable analysis (Table 4).

\section{BCVA 5 years \pm 1 year after first visit}

There were a total of 146 eyes (92 patients) with at least one follow-up visit between 4 and 6 years after the first visit. Among the remaining 71 eyes that were not selected, 45 eyes had a duration of follow-up shorter than 4 years and 26 eyes had a duration of follow-up longer than 6 years.

Comparisons of baseline variables between selected and not selected groups are provided in Supplementary Table 1. The group of selected eyes had a significantly lower proportion of male eyes compared to the group of not selected eyes ( $74 \%$ vs. $87 \%$, respectively; $\mathrm{p}=0.04$ ). There was not enough evidence to conclude that any other baseline variable differed between the two groups.

The most common treatment pattern in the selected group was 'no treatment' ( 82 eyes; $56.2 \%$ ), followed by 1 session of PDT laser (18 eyes; 12.3\%), 1 session of thermal laser ( 8 eyes; 5.5\%), and 2 sessions of PDT laser (3 eyes; 2.1\%). All other eyes received 0-32 sessions of anti-VEGF therapy combined with 0-2 sessions of PDT laser and 0-1 session of thermal laser (35 eyes; $24.0 \%)$.

There was not enough evidence to conclude that mean BCVA at baseline and at 5 years differed ( 0.43 vs. 0.44 , respectively; $p=0.65)$. In the sample, the most common pattern was stable good vision from baseline to 5-year follow-up (35\% of eyes), followed by stable poor vision (18\%), stable intermediate vision (15\%), improvement from intermediate to good vision (14\%), and deterioration from good to intermediate vision (8\%) as shown in Supplementary Table 3. Most eyes with either good or poor initial vision tended to stay in their BCVA group at the 5-year visit 
379 (80\% and $87 \%$, respectively). However, only $42 \%$ of eyes with intermediate initial vision

maintained their BCVA status after 5 years, while $40 \%$ improved and $17 \%$ deteriorated.

Univariable analysis (Table 3 ) revealed that greater age at $1^{\text {st }}$ visit $(\mathrm{p}=0.001)$, greater number of years from disease onset to first visit $(\mathrm{p}<0.0001)$, and PDT laser treatment $(\mathrm{p}=0.04)$ were significantly associated with deterioration of BCVA between initial and 5-year visits. Race was also found to be associated with change in visual acuity $(\mathrm{p}=0.02)$. Specifically, each additional year in age at $1^{\text {st }}$ visit was associated with an increase of $0.008 \log$ MAR (95\%CI: 0.003 to 0.012); each additional year between disease onset and initial visit was associated with an increase of $0.011 \log$ MAR (95\%CI: 0.006 to 0.016$)$. There was not enough evidence to conclude that any other proposed factors were associated with change in BCVA at 5 years. Multivariable analysis (Table 4) revealed that age at $1^{\text {st }}$ visit $(\mathrm{p}=0.03)$, number of years from onset to $1^{\text {st }}$ visit $(\mathrm{p}=0.002)$, and PDT laser treatment $(\mathrm{p}=0.0496)$ were significantly associated with BCVA change. Race was no longer significantly associated with change in BCVA in the multivariable analysis. Otherwise, these results were similar to the univariable analyses

\section{BCVA 10 years \pm 2 years after first visit}

There were a total of 128 eyes (77 patients) with at least one follow-up visit between 8 and 12 years after the first visit. Among the remaining 89 eyes that were not selected, 85 eyes had a duration of follow-up shorter than 8 years and 4 eyes had a duration of follow-up longer than 12 years.

Comparisons of baseline variables between selected and not selected groups are provided in Supplementary Table 1 . Eyes that had a 10 -year follow-up visit were significantly younger at $1^{\text {st }}$ visit compared to eyes that did not (mean age $=57$ vs. 66 years, respectively; $p<0.0001$ ), and were older at disease onset (mean age $=56$ vs. 51 years; $\mathrm{p}=0.04$ ). Also, corticosteroid use at baseline 
402

403

404

405

406

407

408

409

410

411

412

413

414

415

416

417

418

419

420

421

422

423

424

was significantly more prevalent in selected eyes compared to not selected (32\% vs. $17 \%$, respectively; $\mathrm{p}=0.047$ ). There was not enough evidence to conclude that any other baseline variable differed between the two groups.

The most common treatment pattern in this group was 'no treatment' (69 eyes; 53.9\% of eyes), followed by 1 session of PDT laser (11 eyes; 8.6\%), 1 session of thermal laser (6 eyes; 4.7\%), 2 sessions of PDT laser (4 eyes; 3.1\%), and 2 sessions of thermal laser (4 eyes; 3.1\%). All other eyes received 0-75 sessions of anti-VEGF therapy combined with 0-4 sessions of PDT laser and $0-11$ sessions of thermal laser $(26.6 \%)$.

The mean $\operatorname{logMAR}$ BCVA was significantly higher at 10 years follow-up compared to baseline ( 0.46 vs. 0.40 , respectively; $p=0.04$ ). In the sample, the most common pattern was stable good vision from baseline to 10-year follow-up (39\% of eyes), followed by stable poor vision (17\%), improvement from intermediate to good vision (15\%), stable intermediate (10\%), and deterioration from good to intermediate (7\%) and intermediate to poor $(7 \%)$ as shown in Table 5. Most eyes with either good or poor initial vision tended to stay in their BCVA group at the 10year visit (79\% and 92\%, respectively). However, only 32\% of eyes with intermediate initial vision maintained their BCVA status after 10 years, while $46 \%$ improved and $22 \%$ deteriorated. Univariate analysis (Table 3$)$ revealed that greater age at $1^{\text {st }}$ visit $(\mathrm{p}=0.001)$ and number of years from onset to first visit $(\mathrm{p}=0.02)$ were significantly associated with deterioration of BCVA between first and 10-year follow-up visits. Specifically, each additional year in age at $1^{\text {st }}$ visit was associated with an increase of $0.009 \log$ MAR (95\%CI: 0.004 to 0.015$)$, and each additional year between onset and first visit was associated with an increase of $0.010 \operatorname{logMAR}$ (95\%CI: 0.002 to 0.018 ). There was not enough evidence to conclude that any other factors were associated with change in BCVA at 10 years. 
Multivariate analysis (Table 4$)$ revealed that age at $1^{\text {st }}$ visit $(\mathrm{p}=0.001)$ was the only variable associated with BCVA change. Number of years from onset to $1^{\text {st }}$ visit was no longer significantly associated with change in BCVA in the multivariable analysis. Otherwise, these results were similar to univariable analyses.

\section{ASSOCIATION BETWEEN SELECTED VARIABLES AND VISUAL ACUTY AT}

\section{FINAL VISIT}

Univariable analysis of variables measured only at last visit or at unknown time before last visit (Table 6) revealed that CNV ( $\mathrm{p}=0.001)$, FAF change at central fovea $(\mathrm{p}<0.0001)$, cystoid macular degeneration $(\mathrm{p}<0.0001)$, photoreceptor line disruption $(\mathrm{p}<0.0001)$, and external limiting membrane disruption at the fovea $(\mathrm{p}<0.0001)$ were significantly associated with poorer BCVA at last visit. Specifically, BCVA was $0.27 \log$ MAR greater (95\%CI: 0.11 to 0.44 larger) in eyes with CNV at or before last visit, compared to eyes that did not have CNV. Similarly, occurrence of FAF changes at central fovea, cystoid macular degeneration, photoreceptor lines disruption, and external limiting membrane disruption at the fovea were associated with respective $\log$ MAR increases of $0.47,0.56,0.57$, and 0.78 . There was not enough evidence to conclude that subfoveal choroidal thickness at last visit was associated with BCVA at last visit $(\mathrm{p}=0.44)$.

\section{DISCUSSION}

This study reports the visual outcomes and causes of vision loss in chronic CSC. The major findings are as follows: (1) Long-term visual outcomes in chronic CSC are generally favorable and almost $55 \%$ of patients maintain better than 20/40 vision in at least one eye after 10 years of disease; (2) Later age of disease onset is significantly associated with change in BCVA in 
chronic CSC; (3) Cystoid macular degeneration, choroidal NV and disruption to the EZ are associated with poor VA at last visit in chronic CSC; (4) Type $1 \mathrm{NV}$ is the most frequent subtype of NV associated with chronic CSC.

Central serous chorioretinopathy is a relatively common maculopathy that is managed by general ophthalmologists and retina specialists. The incidence varies widely between populations and has been estimated to be $0.0058 \%$ in Olmstead county, Minnesota and $0.21 \%$ in Taiwan. ${ }^{12,35}$ Knowledge concerning long-term visual outcomes and causes of vision loss in chronic CSC are of paramount importance to clinicians yet there are few longitudinal studies that have systematically evaluated these issues. Spaide and colleagues ${ }^{3}$ followed 130 patients with classic and chronic CSC over a mean period of 2.7 years and did not find a difference in mean visual acuity between initial and final visits. Levine et al. ${ }^{5}$ performed a longitudinal study of 14 eyes of 13 patients with acute or chronic CSC for a mean period of 8.2 years using FA and found that 4 eyes lost more than 2 lines on the Snellen acuity chart during the follow-up period. Breukink et al. ${ }^{36}$ performed a retrospective analysis on 52 eyes of 36 patients with chronic CSC and found a mean decline of visual acuity of $0.16 \log$ MAR in 31 eyes without persistent fluid at final visit, after a mean follow-up of 10.6 years. By comparison, in this study we demonstrate a mean BCVA decline from $0.40 \log$ MAR to $0.46 \log$ MAR after 10 years of disease $(\mathrm{p}=0.04)$. However, in the study from Breukink et al, the number of treatments had no effect on visual change, which is consistent with our results. Haga et $\mathrm{al}^{37}$ reported a retrospective analysis of 79 eyes of 73 patients with chronic CSC treated with half-dose PDT and followed-up for at least 3 years. Among these 79 eyes, 64 eyes were considered successful with subretinal fluid reabsorption and without recurrence after 1 PDT session. Multivariate analysis showed that lower baseline BCVA and older age were associated with unsuccessful PDT. ${ }^{37}$ By comparison, in our cohort, PDT 
treatment had no significant effect on BCVA change over a period of 10 years. This may be explained by the inclusion of overall more severe cases in our cohort, longer follow-up or both. We found that a large proportion (79\%) of eyes with good VA at the initial visit (Snellen acuity $20 / 40$ or better) maintained the same level of vision at the 10 -year follow-up visit. Similarly, a significant proportion (92\%) of eyes with poor VA (Snellen acuity 20/200 or worse) at the initial visit remained at the same level of poor vision at the final visit. Patients with intermediate vision between these two levels had a more balanced likelihood of improving vision (46\%), experiencing further visual deterioration (22\%) or remaining stable (32\%). Importantly, $79.7 \%$ of patients in the cohort met the visual standard to qualify for a driver's license at the final visit (VA 20/40 or better in at least 1 eye) and only a small proportion of subjects (12.8\%) were deemed legally blind (20/200 or worse in both eyes) at the final visit. Long-term visual outcomes in chronic CSC therefore appear to compare favorably to neovascular age-related macular degeneration, the most common maculopathy causing severe irreversible vision loss in the developed world. In the Comparison of Age-related Macular Degeneration Treatment Trial (CATT), ${ }^{38} 49.6 \%$ of eyes had 20/40 or better Snellen acuity and 20\% had 20/200 or worse Snellen acuity after 5 years.

In this study, a significant factor associated with poor VA at the final visit was the occurrence of foveal photoreceptor line disruption as seen on SD-OCT. In our cohort, $67.3 \%$ of eyes demonstrated EZ disruption at the final visit and these eyes demonstrated a mean 0.57 logMAR increase in VA compared to those eyes that did not demonstrate EZ disruption. Our findings are consistent with the previous report by Ojima et al. ${ }^{39}$ who studied the photoreceptor layer in eyes with CSC using three-dimensional OCT and found that thinning and defects in foveal photoreceptor IS and OS layers were significantly associated with VA reduction. Spaide and 
Klancnik ${ }^{23}$ evaluated the significant predictors of VA in 30 patients with CSC using covariates derived from FAF and OCT imaging. They found that normalized central macular autofluorescence, the standard deviation of central macular autofluorescence, presence of pigmentary mottling and the presence of subretinal fluid were significantly associated with VA. Imamura et al. ${ }^{21}$ evaluated 475 eyes with CSC (acute and chronic) and correlated FAF abnormalities with VA. With respect to macular FAF changes they found that the occurrence of confluent and granular hypoautofluorescence correlated significantly with decreased VA. In our series, $74.7 \%$ of eyes demonstrated FAF changes at the central fovea. This was another variable strongly associated with poorer VA at the final visit. Taken together, our study demonstrates that structural changes underlying foveal atrophy are the most important anatomic determinants of final VA in chronic CSC. In our study, greater age at disease diagnosis was also found to be significantly associated with change in VA after 5 and 10 years and this finding is consistent with previous reports. ${ }^{21}$ However, a 1 year increase in the age at disease onset was associated with only 0.005 (at 5 years) and 0.009 (at 10 years) increases in logMAR VA, therefore the influence of this variable on VA change is quite small.

Choroidal NV is a recognized complication of CSC with a reported prevalence between 5.8$15.1 \% .^{3,31,40}$ In our study, the prevalence of choroidal NV was almost $25 \%$ and all lesions were classified as type $1 \mathrm{NV}$. Consistent with our work, Fung et al. ${ }^{24}$ and Bonini Filho et al. ${ }^{41}$ also documented an association between type $1 \mathrm{NV}$ and CSC raising the possibility that the anatomic and physiologic alterations inherent to CSC such as choroidal congestion, vascular dilation and subsequent RPE changes may culminate in raised VEGF levels in the sub-RPE compartment. In this study, $59.3 \%$ of the choroidal NV occurred outside the subfoveal region thereby sparing the region of highest visual acuity. Grossnikalus and Green ${ }^{42}$ proposed that type $1 \mathrm{NV}$ may serve to 
nourish the outer retina and RPE. The occurrence of type $1 \mathrm{NV}$ was associated with poorer visual outcomes in this study with eyes demonstrating NV having a mean of 0.27 logMAR increase in BCVA at the final visit compared to eyes without NV. The purpose of this study was not to evaluate the response of choroidal NV to various forms of therapy however statistical analysis did not reveal a significant association between mode of treatment for choroidal NV and visual change at the 10-year follow-up visit. This may be due to the limited sample size of the study.

Corticosteroid use is an important risk factor for the development of CSC however its influence on visual outcomes remains unclear. In the prospective study by Carvalho-Recchia et al., ${ }^{11} 52 \%$ of cases of CSC had a history of steroid use within 1 month of onset of symptoms while the frequency of steroid use in age- and sex-matched controls was only $16 \%$. Male gender is another known risk factor for CSC with a male/female incidence ratio estimated to be 1.74 in Taiwan. ${ }^{9}$ Visual outcomes in female patients with CSC are known to be favorable with studies by Perkins et al. ${ }^{43}$ and Quillen et al. ${ }^{44}$ demonstrating that $88 \%$ and $78 \%$, respectively, of female subjects with active CSC achieve a final VA of 20/40 or better. However, few studies have compared visual outcomes between male and female patients with chronic CSC in the same population. In this study, gender and corticosteroid use were not significantly correlated with visual outcomes. Our findings exemplify the complex etiopathogenic mechanisms underlying CSC and suggest that the risk factors for the development of CSC may be incongruous with the pathogenic factors that modulate the natural course of this disease and long-term visual outcomes.

We acknowledge that large multi-center trials with standardized follow up intervals are the gold standard technique for studying the influence of a disease process and intervention on visual 
outcomes. There are several limitations of this study including its retrospective design and the non-standardized manner by which patients were reviewed throughout their disease course. This study included patients that were managed by 3 retina specialists between the period of 1977 and 2017. As OCT and fundus autofluorescence imaging were widely employed in clinical ophthalmology only after the year 2005, multimodal imaging was not available to assist in the management of many patients in our cohort during the early part of their disease course. For this reason it was not possible to precisely determine when CME, CNV and EZ disruption occurred in a significant number of patients; i.e. those seen before the year 2000. We acknowledge that this can only be determined using a prospectively-designed study where multimodal imaging is performed at each visit. However, as all patients in our cohort had at least one visit where OCT and fundus autofluorescence imaging were contemporaneously performed we were able to investigate the association between changes seen on these multimodal imaging devices and final VA. We feel that this is still an important finding and provides new information that can be used to design future studies to investigate the exact timing of CNV, CME and EZ disruption during the natural course of CSC.

There may be a selection bias due to the referral nature of complex cases to our practice. Our results may not reflect the visual prognosis of chronic central serous chorioretinopathy in the general community and be overall more severe. Additionally, it was not possible to reliably quantify the number of recurrent episodes of PED and neurosensory detachment in each eye therefore, similar to other reports, our definition of chronic CSC was based on the occurrence of persistent subretinal fluid. ${ }^{9}$ It is plausible that the number of recurrent episodes correlates with visual outcomes in CSC and prospective studies investigating this hypothesis are warranted. Moreover, the numbers of years from disease onset to $1^{\text {st }}$ visit was found to be a significant 


\section{ACKNOWLEDGEMENTS}

$580 \quad$ Nil of long-term visual outcomes.

determinant of the change in visual acuity in this report and there may be some error in patient report of the age of onset. But this suggests that the time between the beginning of visual symptoms and first visit in a specialized center was correlated with visual prognosis and our results plead for earlier management in a specialized center, as has been suggested by other authors. ${ }^{37}$ Whether the disease starts earlier or later, the visual prognosis appeared to be more correlated with the duration of the disease than with the age of onset. This suggests that some factors influencing the visual prognosis may be due to aging. If there is an age-related retinal degenerative process involved in the visual loss in CSC, this would raise the question of some overlap in the biological pathways involved in age-related macular degeneration and chronic CSC with central outer retinal and RPE atrophy.

Despite the retrospective nature of our data we propose that our study provides important and new clinical information regarding the long-term outcomes of chronic CSC that has major relevance for clinical practice. Major strengths of this study include a relatively large sample size, a prolonged duration of follow up, the application of state-of-the-art multimodal imaging to define disease phenotype and robust statistical techniques to determine the significant predictors 
Figure 1 - Choroidal neovascularization (NV) due to chronic central serous chorioretinopathy.

585

586

587
Multimodal imaging findings of type $1 \mathrm{NV}$ in a 53-year-old male from a single visit are presented. Color imaging (A) reveals exudation overlying the area of NV. Fundus autofluorescence (FAF) imaging demonstrates subretinal fluid and a gravitating neurosensory detachment (B). Fluorescein angiography (FA; C and D) reveals leakage that is mostly clearly seen in the late frames. Indocyanine green angiography (ICGA; E and F) demonstrates a hyperfluroescent plaque (arrowhead) at the site of NV that is also best seen in the late frames. A pigment epithelial detachment is visualized using spectral-domain optical coherence tomography (G) and hyperreflective material beneath the retinal pigment epithelium correlating to type $1 \mathrm{NV}$ (arrow) is evident. The region from which the B scan image was acquired is presented on the color photo (white line).

Figure 2 - Fundus autofluorescence (FAF) imaging patterns in chronic central serous chorioretinopathy. Eyes in this study demonstrated a spectrum of FAF alterations including mild changes involving the posterior pole and peripapillary region (A), multifocal lesions (B), gravitating tracts $(\mathrm{C})$ and diffuse areas of FAF change that correlated to sites of previous bullous retinal detachment (D). Granular (E) or confluent (F) FAF changes were also seen in the fovea in $74.9 \%$ of eyes.

Figure 3 - Cystoid macular degeneration (CMD) due to chronic central serous chorioretinopathy. Multimodal imaging findings of CMD in a 66-year-old male from a single visit are presented. Color (A) and fundus autofluorescence (FAF) imaging (B) reveals atrophy and attenuation of the retinal pigment epithelium at the site of CMD. There was no leakage within these regions on fluorescein angiography (FA; C) and indocyanine green angiography 
606 (ICGA; D). Spectral-domain optical coherence tomography (E) demonstrates intraretinal cysts 607 and disorganization of outer retinal layers. The region from which the B scan image was 608 acquired is presented on the color photo (white line). 
610

611

612

613

614

615

616

617

618

619

620

621

622

623

624

625

626

627

628

629

630

631

632

633

\section{REFERENCES}

1. Daruich A, Matet A, Dirani A, et al. Central serous chorioretinopathy: Recent findings and new physiopathology hypothesis. Prog Retin Eye Res. 2015;48:82-118.

2. Haimovici R, Koh S, Gagnon DR, et al. Risk factors for central serous chorioretinopathy: a case-control study. Ophthalmology. 2004;111:244-249.

3. Spaide RF, Campeas L, Haas A, et al. Central serous chorioretinopathy in younger and older adults. Ophthalmology. 1996;103:2070-2079; discussion 2079-2080.

4. Gilbert CM, Owens SL, Smith PD, Fine SL. Long-term follow-up of central serous chorioretinopathy. Br J Ophthalmol. 1984;68:815-820.

5. Levine R, Brucker AJ, Robinson F. Long-term follow-up of idiopathic central serous chorioretinopathy by fluorescein angiography. Ophthalmology. 1989;96:854-859.

6. Loo RH, Scott IU, Flynn HW, Jr., et al. Factors associated with reduced visual acuity during long-term follow-up of patients with idiopathic central serous chorioretinopathy. Retina. 2002;22:19-24.

7. Bujarborua D. Long-term follow-up of idiopathic central serous chorioretinopathy without laser. Acta Ophthalmol Scand. 2001;79:417-421.

8. Mrejen S, Sarraf D, Mukkamala SK, Freund KB. Multimodal imaging of pigment epithelial detachment: a guide to evaluation. Retina. 2013;33:1735-1762.

9. Bae SH, Heo JW, Kim C, et al. A randomized pilot study of low-fluence photodynamic therapy versus intravitreal ranibizumab for chronic central serous chorioretinopathy. Am J Ophthalmol. 2011;152:784-792 e782.

10. Fujimoto H, Gomi F, Wakabayashi T, et al. Morphologic changes in acute central serous chorioretinopathy evaluated by fourier-domain optical coherence tomography. Ophthalmology. 2008;115:1494-1500, 1500 e1491-1492. 
649

650

651

652

11. Carvalho-Recchia CA, Yannuzzi LA, Negrao S, et al. Corticosteroids and central serous chorioretinopathy. Ophthalmology. 2002;109:1834-1837.

12. Tsai DC, Chen SJ, Huang CC, et al. Epidemiology of idiopathic central serous chorioretinopathy in Taiwan, 2001-2006: a population-based study. PLoS One. 2013;8:e66858. 13. Yannuzzi LA. Type A behavior and central serous chorioretinopathy. Trans Am Ophthalmol Soc. 1986;84:799-845.

14. Hirami Y, Tsujikawa A, Sasahara M, et al. Alterations of retinal pigment epithelium in central serous chorioretinopathy. Clin Exp Ophthalmol. 2007;35:225-230.

15. Mudvari SS, Goff MJ, Fu AD, et al. The natural history of pigment epithelial detachment associated with central serous chorioretinopathy. Retina. 2007;27:1168-1173.

16. Dansingani KK, Balaratnasingam C, Naysan J, Freund KB. En Face Imaging of Pachychoroid Spectrum Disorders with Swept-Source Optical Coherence Tomography. Retina. 2016;36:499-516.

17. Imamura Y, Fujiwara T, Margolis R, Spaide RF. Enhanced depth imaging optical coherence tomography of the choroid in central serous chorioretinopathy. Retina. 2009;29:14691473.

18. Yannuzzi LA, Shakin JL, Fisher YL, Altomonte MA. Peripheral retinal detachments and retinal pigment epithelial atrophic tracts secondary to central serous pigment epitheliopathy. Ophthalmology. 1984;91:1554-1572.

19. Balaratnasingam C, Freund KB, Tan AM, et al. Bullous Variant of Central Serous Chorioretinopathy: Expansion of Phenotypic Features Using Multimethod Imaging. Ophthalmology. 2016;123:1541-1552. 
657

658

659

660

661

662

663

664

665

666

667

668

669

670

671

672

673

674

675

676

677

678

Forms in Chronic Central Serous Chorioretinopathy. Am J Ophthalmol. 2016;166:60-67.

21. Imamura Y, Fujiwara T, Spaide RF. Fundus autofluorescence and visual acuity in central serous chorioretinopathy. Ophthalmology. 2011;118:700-705.

22. Ober MD, Yannuzzi LA, Do DV, et al. Photodynamic therapy for focal retinal pigment epithelial leaks secondary to central serous chorioretinopathy. Ophthalmology. 2005;112:20882094.

23. Spaide RF, Klancnik JM, Jr. Fundus autofluorescence and central serous chorioretinopathy. Ophthalmology. 2005;112:825-833.

24. Fung AT, Yannuzzi LA, Freund KB. Type 1 (sub-retinal pigment epithelial)

neovascularization in central serous chorioretinopathy masquerading as neovascular age-related macular degeneration. Retina. 2012;32:1829-1837.

25. Iida T, Yannuzzi LA, Spaide RF, et al. Cystoid macular degeneration in chronic central serous chorioretinopathy. Retina. 2003;23:1-7; quiz 137-138.

26. Pang CE, Freund KB. Pachychoroid neovasculopathy. Retina. 2015;35:1-9.

27. Freund KB, Ho IV, Barbazetto IA, et al. Type 3 neovascularization: the expanded spectrum of retinal angiomatous proliferation. Retina. 2008;28:201-211.

28. Freund KB, Zweifel SA, Engelbert M. Do we need a new classification for choroidal neovascularization in age-related macular degeneration? Retina. 2010;30:1333-1349.

29. Gass DM. Atlas of Macular Diseases. Agarwal A, editor: Saunders ; 2012.

30. Subfoveal neovascular lesions in age-related macular degeneration. Guidelines for evaluation and treatment in the macular photocoagulation study. Macular Photocoagulation Study Group. Arch Ophthalmol. 1991;109:1242-1257. 
679

680

681

682

683

684

685

686

687

688

689

690

691

692

693

694

695

696

697

698

699

31. Hage R, Mrejen S, Krivosic V, et al. Flat irregular retinal pigment epithelium detachments in chronic central serous chorioretinopathy and choroidal neovascularization. Am J Ophthalmol. 2015;159:890-903 e893.

32. Framme C, Walter A, Gabler B, et al. Fundus autofluorescence in acute and chronicrecurrent central serous chorioretinopathy. Acta Ophthalmol Scand. 2005;83:161-167.

33. Ying GS, Maguire MG, Glynn R, Rosner B. Tutorial on Biostatistics: Linear Regression Analysis of Continuous Correlated Eye Data. Ophthalmic Epidemiol. 2017;24:130-140.

34. Hosmer DW, Lemeshow S. Applied Logistic Regression. New York: Wiley; 2013.

35. Kitzmann AS, Pulido JS, Diehl NN, et al. The incidence of central serous chorioretinopathy in Olmsted County, Minnesota, 1980-2002. Ophthalmology. 2008;115:169173.

36. Breukink MB, Dingemans AJ, den Hollander AI, et al. Chronic central serous chorioretinopathy: long-term follow-up and vision-related quality of life. Clin Ophthalmol. 2017;11:39-46.

37. Haga F, Maruko R, Sato C, et al. Long-term prognostic factors of chronic central serous chorioretinopathy after half-dose photodynamic therapy: A 3-year follow-up study. PLoS One. 2017;12:e0181479.

38. Comparison of Age-related Macular Degeneration Treatments Trials Research G, Maguire MG, Martin DF, et al. Five-Year Outcomes with Anti-Vascular Endothelial Growth Factor Treatment of Neovascular Age-Related Macular Degeneration: The Comparison of AgeRelated Macular Degeneration Treatments Trials. Ophthalmology. 2016;123:1751-1761. 
39. Ojima Y, Hangai M, Sasahara M, et al. Three-dimensional imaging of the foveal photoreceptor layer in central serous chorioretinopathy using high-speed optical coherence tomography. Ophthalmology. 2007;114:2197-2207.

40. Peiretti E, Ferrara DC, Caminiti G, et al. Choroidal Neovascularization in Caucasian Patients with Longstanding Central Serous Chorioretinopathy. Retina. 2015;35:1360-1367. 41. Bonini Filho MA, de Carlo TE, Ferrara D, et al. Association of Choroidal Neovascularization and Central Serous Chorioretinopathy With Optical Coherence Tomography Angiography. JAMA Ophthalmol. 2015;133:899-906.

42. Grossniklaus HE, Green WR. Choroidal neovascularization. Am J Ophthalmol. 2004;137:496-503.

43. Perkins SL, Kim JE, Pollack JS, Merrill PT. Clinical characteristics of central serous chorioretinopathy in women. Ophthalmology. 2002;109:262-266.

44. Quillen DA, Gass DM, Brod RD, et al. Central serous chorioretinopathy in women. Ophthalmology. 1996;103:72-79. 
A

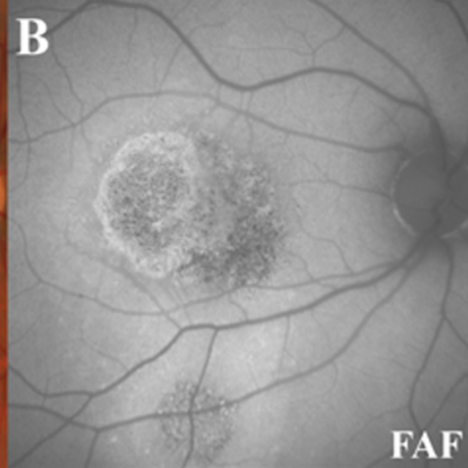

C

FA

D

E

F

FA

0:44

5:47

ICGA

ICGA

0:41

32:3
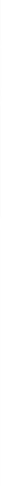

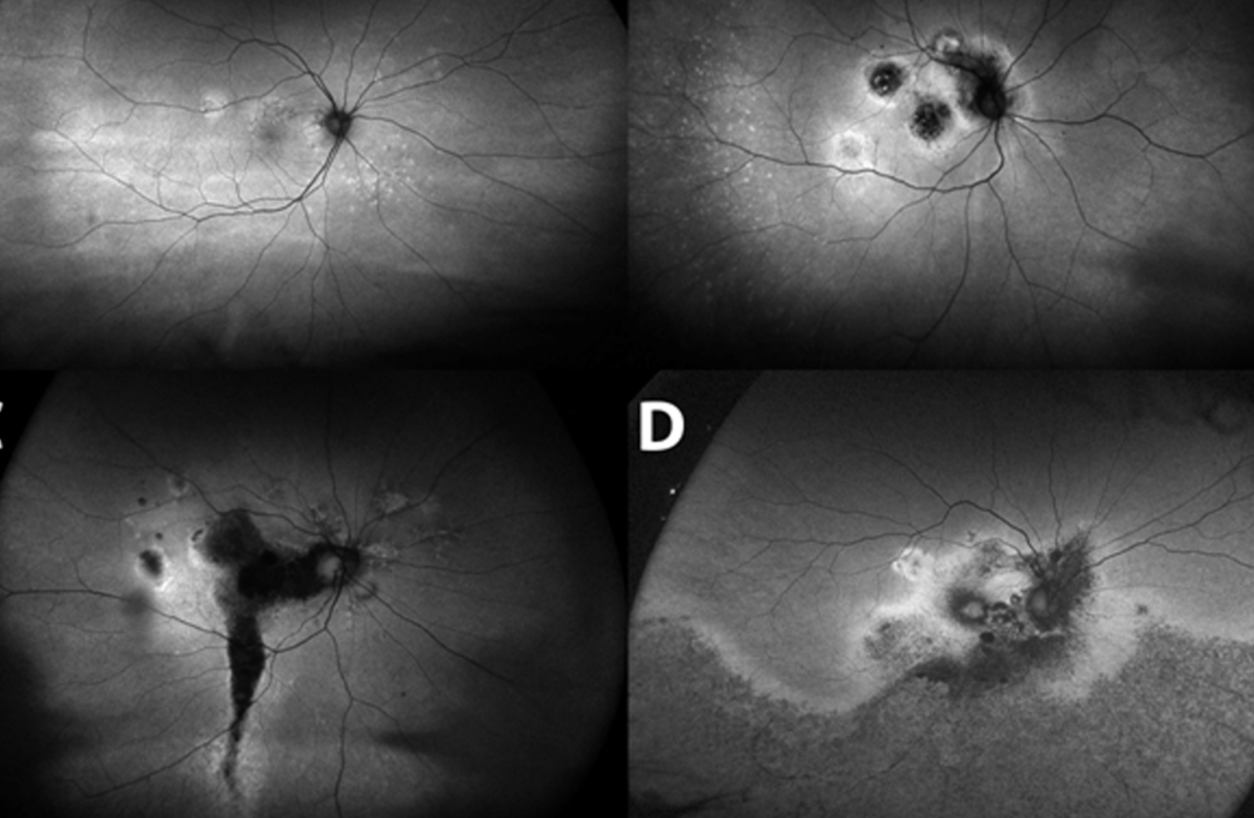

E

F

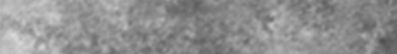

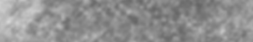

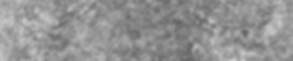

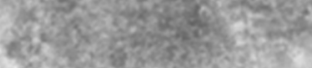

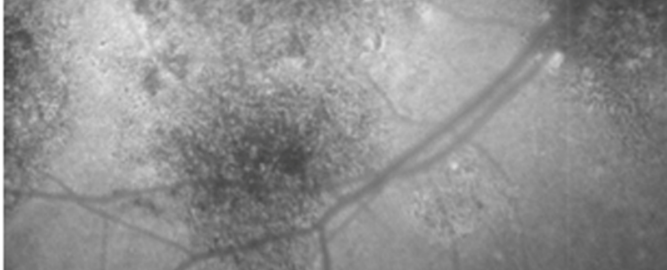

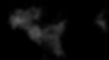

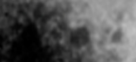

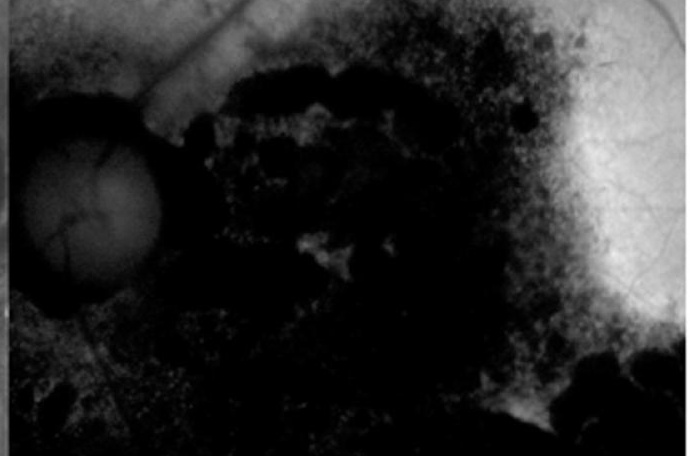

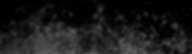

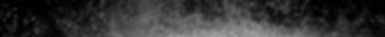




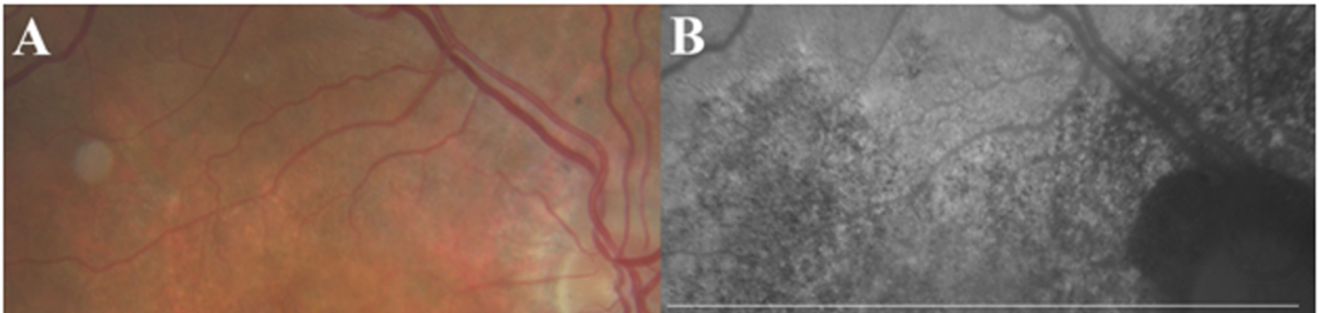

C

D

$\underset{5: 55}{\text { FA }}$

ICGA

1:43

E

\begin{abstract}
se
\end{abstract}
8

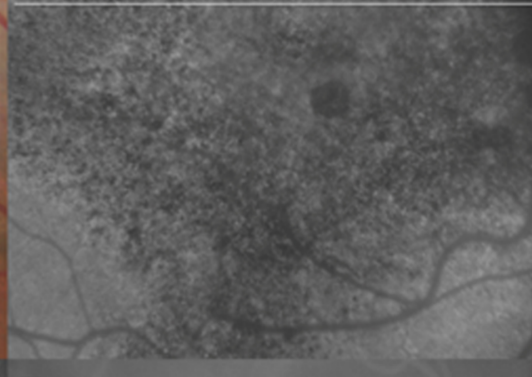

FAF

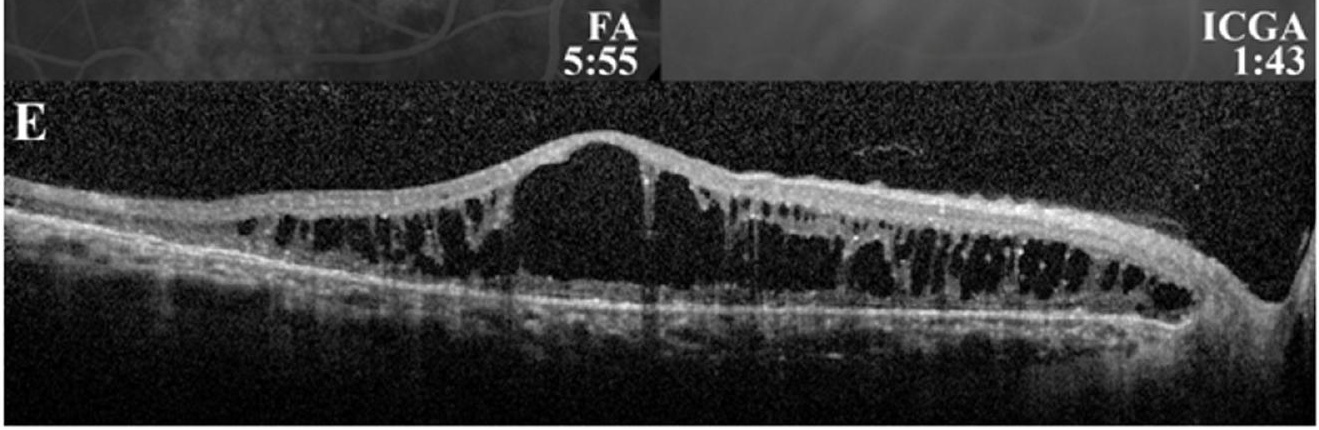




\section{Total or Mean}

\section{CHOROID}

Subfoveal choroidal thickness ( $\mu \mathrm{m})$

397.4

Neovascularization (any subtype)

$52(23.9 \%)$

type $1 \mathrm{NV}$

$52(100.0 \%)$

type $2 \mathrm{NV}$

$0(0.0 \%)$

type $3 \mathrm{NV}$

$0(0.0 \%)$

RPE

Hypoautofluorescence in any region

$217(100 \%)$

Hypoautofluorescence in fovea

$162(74.7 \%)$

Gravitating tracts

$105(48.4 \%)$

\section{RETINA}

Subretinal fibrosis

$25(11.5 \%)$

Disruption to EZ

$90(41.5 \%)$

Disruption to ELM

$146(67.3 \%)$

Cystoid macular degeneration

$47(21.7 \%)$

Central subfield thickness $(\mu \mathrm{m})$

$436.8 \pm 257.6$

Point foveal thickness ( $\mu \mathrm{m})$

$451.4 \pm 420.1$

Table 2 - Summary of Choroid, RPE and retinal structural alterations.

$\mathrm{ELM}=$ External limiting membrane; EZ = Ellipsoid Zone. 


\begin{tabular}{|c|c|c|c|c|c|c|}
\hline \multirow[b]{2}{*}{ Baseline variables } & \multicolumn{2}{|c|}{$\begin{array}{l}\text { 1-YEAR FOLLOW-UP } \\
(\mathrm{N}=131)\end{array}$} & \multicolumn{2}{|c|}{$\begin{array}{c}\text { 5-YEAR FOLLOW-UP } \\
(\mathrm{N}=146)\end{array}$} & \multicolumn{2}{|c|}{$\begin{array}{l}\text { 10-YEAR FOLLOW-UP } \\
\qquad(\mathrm{N}=128)\end{array}$} \\
\hline & $\begin{array}{l}\text { Beta coefficient }(95 \% \\
\text { confidence interval) }\end{array}$ & p-value & $\begin{array}{c}\text { Beta coefficient }(95 \% \\
\text { confidence interval) }\end{array}$ & $\mathrm{p}$-value & $\begin{array}{c}\text { Beta coefficient }(95 \% \\
\text { confidence interval) }\end{array}$ & $\mathrm{p}$-value \\
\hline Age at $1^{\text {st }}$ visit & $0.005(-0.000,0.010)$ & 0.05 & $0.008(0.003,0.012)$ & 0.001 & $0.009(0.004,0.015)$ & 0.001 \\
\hline Age at onset & $-0.002(-0.006,0.003)$ & 0.44 & $-0.000(-0.004,0.004)$ & 1 & $0.003(-0.002,0.008)$ & 0.21 \\
\hline $\begin{array}{l}\text { Number of years from } \\
\text { onset to } 1^{\text {st }} \text { visit }\end{array}$ & $0.007(0.002,0.011)$ & 0.01 & $0.011(0.006,0.016)$ & $<0.0001$ & $0.010(0.002,0.018)$ & 0.02 \\
\hline Bilateral disease & $0.12(-0.00,0.26)$ & 0.06 & $0.06(-0.08,0.19)$ & 0.41 & $0.12(-0.04,0.29)$ & 0.14 \\
\hline Left eye & $-0.09(-0.17,-0.01)$ & 0.04 & $-0.04(-0.13,0.06)$ & 0.42 & $-0.04(-0.16,0.08)$ & 0.56 \\
\hline Gender (Male) & $0.08(-0.05,0.21)$ & 0.21 & $0.03(-0.10,0.16)$ & 0.68 & $0.01(-0.14,0.15)$ & 0.91 \\
\hline Race & & 0.81 & & 0.02 & & 0.21 \\
\hline Smoking & $-0.06(-0.19,0.06)$ & 0.31 & $-0.06(-0.21,0.08)$ & 0.39 & $-0.05(-0.23,0.13)$ & 0.58 \\
\hline$H T N$ & $-0.00(-0.11,0.11)$ & 0.99 & $0.05(-0.07,0.17)$ & 0.45 & $0.03(-0.11,0.16)$ & 0.7 \\
\hline$D M$ & $0.02(-0.16,0.19)$ & 0.85 & $0.15(-0.05,0.35)$ & 0.13 & $0.07(-0.17,0.30)$ & 0.57 \\
\hline Steroid use & $0.01(-0.10,0.13)$ & 0.8 & $0.01(-0.11,0.13)$ & 0.88 & $0.04(-0.10,0.17)$ & 0.58 \\
\hline Thermal laser* & $-0.07(-0.29,0.15)$ & 0.53 & $0.08(-0.10,0.26)$ & 0.36 & $0.11(-0.07,0.29)$ & 0.24 \\
\hline PDT laser* & $0.04(-0.07,0.15)$ & 0.49 & $0.12(0.01,0.24)$ & 0.04 & $-0.02(-0.16,0.13)$ & 0.83 \\
\hline Anti-VEGF therapy* & $0.07(-0.06,0.21)$ & 0.28 & $0.10(-0.04,0.24)$ & 0.14 & $0.10(-0.05,0.25)$ & 0.17 \\
\hline
\end{tabular}

Table 3: Univariate analysis of factors associated with BCVA change.

*At least one treatment session between baseline and the visit prior to the 1-year, 5-year, or 10-year visit. 



\begin{tabular}{|c|c|c|}
\hline \multicolumn{3}{|c|}{1 Year Follow-Up $(\mathrm{N}=131)$} \\
\hline Baseline variables & $\begin{array}{c}\text { Beta coefficient (95\% } \\
\text { confidence interval) }\end{array}$ & p-value \\
\hline $\begin{array}{l}\text { Number of years from onset } \\
\text { to } 1^{\text {st }} \text { visit }\end{array}$ & $0.007(0.002,0.012)$ & 0.01 \\
\hline Left eye & $-0.09(-0.17,-0.01)$ & 0.03 \\
\hline \multicolumn{3}{|c|}{5 Year Follow-Up $(N=146)$} \\
\hline Baseline variables & $\begin{array}{l}\text { Beta coefficient (95\% } \\
\text { confidence interval) }\end{array}$ & p-value \\
\hline Age at $1^{\text {st }}$ visit & $0.005(0.001,0.010)$ & 0.03 \\
\hline $\begin{array}{l}\text { Number of years from onset } \\
\text { to } 1^{\text {st }} \text { visit }\end{array}$ & $0.009(0.003,0.014)$ & 0.002 \\
\hline PDT laser treatment* & $0.10(0.00,0.21)$ & 0.0496 \\
\hline \multicolumn{3}{|c|}{10 Year Follow-Up $(N=128)$} \\
\hline Baseline variable & $\begin{array}{c}\text { Beta coefficient (95\% } \\
\text { confidence interval) }\end{array}$ & p-value \\
\hline Age at $1^{\text {st }}$ visit & $0.009(0.004,0.015)$ & 0.001 \\
\hline
\end{tabular}

Table 4: Multivariate analysis of factors associated with BCVA change at different

follow-up time points. *At least one treatment session between baseline and the visit prior to the 5-year visit. 


\begin{tabular}{|c|c|c|c|c|c|}
\hline & & \multicolumn{3}{|c|}{ BCVA at 10 year follow-up } & \multirow[b]{2}{*}{ Total } \\
\hline & & $\begin{array}{c}\operatorname{logMAR} \leq \\
0.3\end{array}$ & $\begin{array}{c}0.3<\operatorname{logMAR}< \\
1\end{array}$ & $\log M A R \geq 1$ & \\
\hline \multirow{4}{*}{ 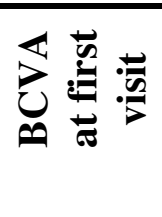 } & $\operatorname{logMAR} \leq 0.3$ & $50(39.06)$ & $9(7.03)$ & $4(3.13)$ & $63(49.22)$ \\
\hline & $0.3<\log$ MAR $<1$ & $19(14.84)$ & $13(10.16)$ & $9(7.03)$ & $41(32.03)$ \\
\hline & $\log M A R \geq 1$ & $1(0.78)$ & $1(0.78)$ & $22(17.19)$ & $24(18.75)$ \\
\hline & Total & $70(54.69)$ & $23(17.97)$ & $35(27.34)$ & $128(100)$ \\
\hline
\end{tabular}

Table 5 - Frequency of vision changes between first visit and 10 year follow-up.

Data are reported as count (percent of total). 